\title{
Cervical Epidural Rhabdomyosarcoma with a Leukemia-like Presentation in an Aged Patient
}

\author{
-Case Report-
}

\author{
Toshihiko HAISA, Tatsuya KONDO, Akiyoshi MIWA*, and Kiyoshi SAITOH** \\ Departments of Neurosurgery, *Hematology, and ${ }^{* *}$ Pathology, \\ International Medical Center of Japan, Tokyo
}

\begin{abstract}
A 77-year-old female presented with rhabdomyosarcoma manifesting as leukemia-like indications. Neuroimagings detected cervical and paravertebral masses. Immunohistochemical study of the surgically excised mass lesion from the cervical spine established the correct diagnosis. This leukemia-like presentation of rhabdomyosarcoma requires a multidisciplinary approach to establish the correct diagnosis and treatment.
\end{abstract}

Key words: rhabdomyosarcoma, leukemia, bone marrow metastasis, immunohistochemistry

\section{Introduction}

Rhabdomyosarcoma is the most common soft tissue sarcoma in infancy, childhood, and adolescence, but is quite rare in the aged. ${ }^{8)}$ Rhabdomyosarcoma frequently metastasizes to the bone marrow ${ }^{8)}$ but bone marrow involvement is rare at the onset of the disease. ${ }^{16)}$ Massive bone marrow infiltration which would be mistaken for acute leukemia is relatively uncommon, as only 30 of 500 cases $(6 \%)$ of rhabdomyosarcoma in children and adolescents had bone marrow involvement at diagnosis. ${ }^{16]}$ However, a number of unusual cases were initially diagnosed as acute leukemia because of similar clinical symptoms and signs and/or because of the presence of 'leukemic' blasts in the bone marrow. ${ }^{1-7,9-15)}$ This unusual presentation is important, because the recognition of rhabdomyoblasts in bone marrow smears is not easy, ${ }^{5)}$ and the treatment and prognosis of rhabdomyosarcoma are different from those of acute leukemia or lymphoma. ${ }^{4)}$

We report a 77-year-old female who presented as acute leukemia, supported by the presence of 'blastoid' cells in the bone marrow aspirate, but was finally diagnosed as having rhabdomyosarcoma based on immunostaining of a surgically excised

Received July 15, 1998; Accepted October 26, 1998 mass lesion from the cervical spine.

\section{Case Report}

A 77-year-old female was seen at a university hospital because of malaise and arthritic pain persisting for 3 weeks and left shoulder pain for one week. The diagnosis was acute leukemia based on laboratory data and a bone marrow aspirate. She was then referred to our center for further evaluation and treatment.

On admission, she complained of severe pain extending from the left shoulder to the hand. General physical examination found the patient was pale without lymphadenopathy or hepato-splenomegaly. Neurological examination revealed left-sided slight hemiparesis and hypesthesia in the left C-6 and C-7 areas with no pathological reflexes.

The blood cell count showed hemoglobin $8.0 \mathrm{~g} / \mathrm{dl}$, hematocrit $25.7 \%$, white cell count $5300 / \mathrm{mm}^{3}$, and a platelet count of $217,000 / \mathrm{mm}^{3}$. Differential white cell count showed $41 \%$ segs, $6 \%$ bands, $35 \%$ lymphocytes, $15 \%$ monocytes, $1 \%$ basophils, $1 \%$ myelocytes, and $1 \%$ atypical cells. Prothrombin time and partial thromboplastin time were normal. Plasma calcium was normal. Bone marrow aspiration performed the day after admission showed infiltration by large blast-like cells with basophilic cytoplasm 


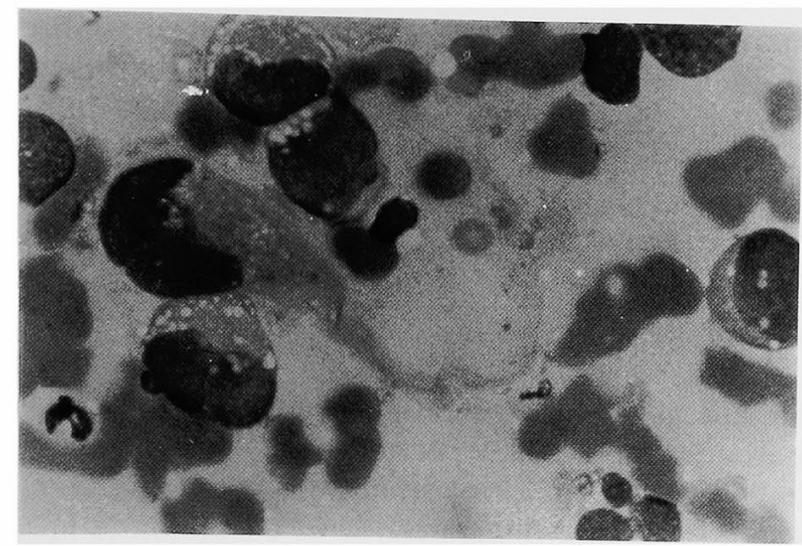

Fig. 1 Photomicrograph of the bone marrow aspirate, showing infiltration of blast-like cells with basophilic cytoplasm and conspicuous cytoplasmic vacuolation, but only a few atypical immature cells with a cytoplasmic filament-like structure. Wright's stain, original magnification $\times 1000$.

and conspicuous cytoplasmic vacuolation, suggestive of acute lymphoblastic leukemia or lymphoma, but meticulous observation found only a few atypical immature cells with a filament-like structure in the cytoplasm (Fig. 1).

Chest radiography showed an anterior mediastinal mass. Computed tomography (CT) and magnetic resonance imaging of the cervical spine disclosed a posterior epidural mass with no osteolytic lesion between C-5 and C-7 (Fig. 2), and CT of the body demonstrated a paravertebral mass at T-12. Thorough investigation detected no other mass lesions.

With the patient in the prone position, the tumor was subtotally excised following C5-7 laminectomy and partial facetectomy on the left. The tumor was solid, yellowish in color, and firmly attached to the dura mater. Histological examination of the excised tissue revealed that the tumor was composed largely of round to oval immature cells and a few large cells with ample eosinophilic cytoplasm (Fig. 3 upper), showing an alveolar pattern (Fig. 3 lower). Immunohistochemical examination showed that the tumor cells were positively stained for muscle-specific actin (Fig. 3 upper inset) and weakly stained for vimentin, but not for leukocyte common antigen, Tcell antigen, B-cell antigen, neuron-specific enolase, epithelial membrane antigen, and cytokeratin. The histological diagnosis was an alveolar type of rhabdomyosarcoma.

The severe pain extending from the left shoulder to the hand disappeared immediately after surgery.

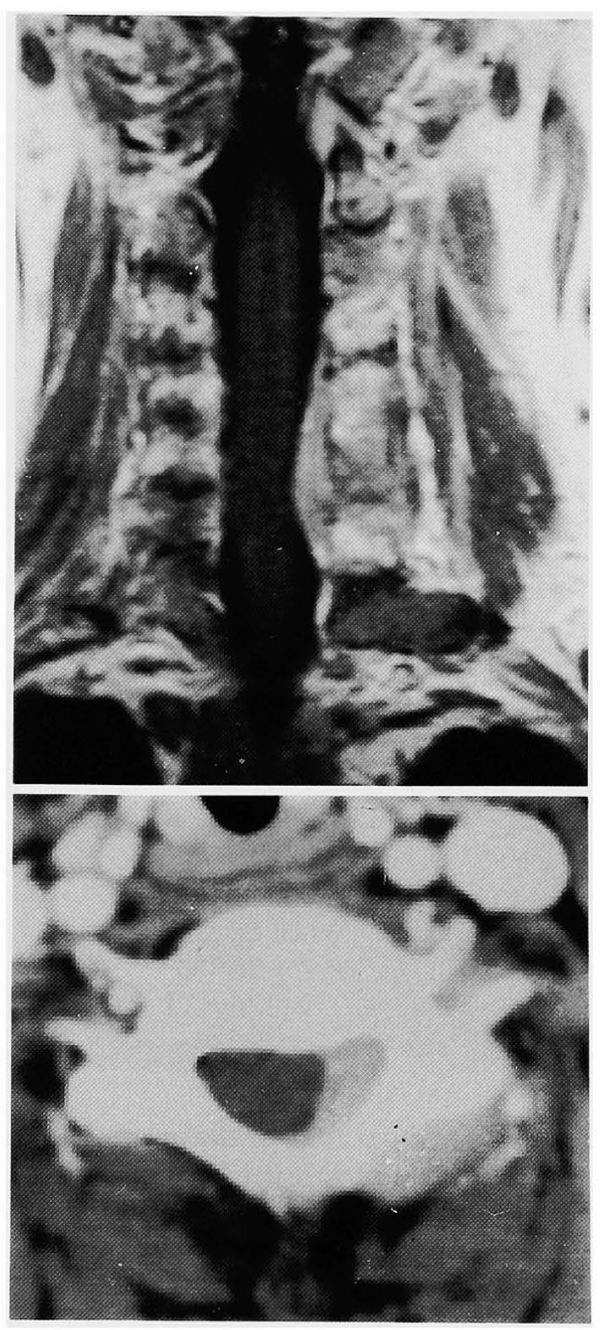

Fig. 2 upper: Coronal magnetic resonance image after gadolinium infusion showing an epidural mass lesion between C-5 and C-7. lower: Axial computed tomography scan with contrast medium showing an enhanced posterior epidural mass lesion extending over the lamina.

The patient received chemotherapy with vincristine, adriamycin, and cyclophosphamide, and partial remission has been achieved.

\section{Discussion}

The classic case of rhabdomyosarcoma with a leukemia-like presentation occurred in a 16-year-old male with malaise, fever, and arthritic symptoms. ${ }^{3)}$ Following the development of anemia, a bone marrow examination showed infiltration by malignant cells leading to a diagnosis of diffuse lymphoma of undefined cell type. Subsequently, skeletal 


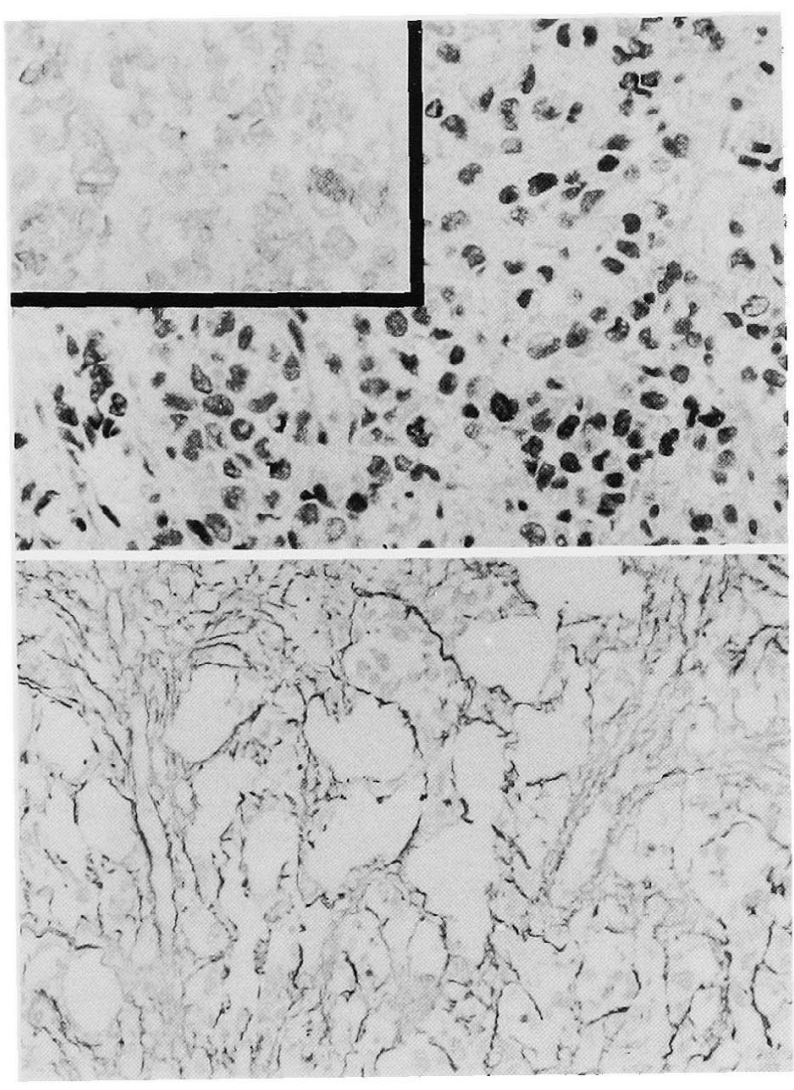

Fig. 3 Photomicrographs of the surgical specimen. upper: Round to oval immature cells and a few large cells with ample eosinophilic cytoplasm. HE stain, original magnification $\times 70$. upper inset: Tumor cells displaying immunoreactivity for musclespecific actin. Original magnification $\times$ 70. lower: Reticulin silver stain showing an alveolar pattern. Original magnification $\times 10$.

radiography demonstrated osteolytic lesions. Postmortem examination confirmed the diagnosis of embryonal rhabdomyosarcoma metastasizing to the bones from an undetermined primary site. More recently, an 18-year-old female presented with easy bruising and fatigue. ${ }^{13)}$ The bone marrow aspirate revealed diffuse involvement by undifferentiated blasts similar to those observed in acute lymphoid leukemia, but intensive studies found no mass or osteolytic lesions. The diagnosis of rhabdomyosarcoma of unknown primary site was based on immunohistochemical study.

In our case, acute leukemia was the initial diagnosis at the referring hospital, and lymphoma was tentatively suspected at our center. Immunohistochemical study of the surgical specimen indicated the correct diagnosis. Immunohistochemical staining with antibodies against desmin, musclespecific actin, and myoglobin are considered to be most useful for diagnostic purposes. ${ }^{2,8)}$ In addition, ultrastructural study is also useful to establish the correct diagnosis. ${ }^{12,14)}$ Some of the characteristic features are distinct bundles of thick (myosin) filaments, alternating thin (actin) and thick (myosin) filaments, or distinct sarcomeres. ${ }^{8)}$

Our experience confirms that rhabdomyosarcoma should be included in the differential diagnosis of patients presenting with mass lesions and blast-like cells in the bone marrow mimicking those seen in acute leukemia. A high index of suspicion is necessary, especially in patients with a leukemia-like presentation and an occult primary site. Rhabdomyoblasts can be mistaken for leukemic blasts or high-grade lymphoma cells, so an experienced observer should review such cases. ${ }^{5}$ Such an initial presentation of rhabdomyosarcoma may be more common than previously recognized, since a misdiagnosis of hematopoietic tumor is easily made. ${ }^{4)}$

Neurosurgeons and hematologists should be aware that the initial manifestation of rhabdomyosarcoma may mimic that of acute leukemia, possibly leading to an inappropriate diagnosis and treatment. The multidisciplinary approach is important for a correct diagnosis.

\section{References}

1) Almanaseer IY, Prinzon T, Taxy JB, Okuno T: Systemic rhabdomyosarcoma with diffuse bone marrow involvement. Case report of an unusual presentation. Am J Clin Pathol 82: 349-353, 1984

2) Ambrosiani L, Bellone S, Betto FS, Cecchetti G, Ceretti E, Dagani R, Morelli A, Pavia G, Schiaffino E, Tavani E, Vismara A: Rhabdomyosarcoma presenting as acute hematologic malignancy: case report and review of the literature. Tumori 82: 408-412, 1996

3) Castleman B, McNeely BU: Case records of Massachusetts General Hospital. Case 4-1972. N Engl J Med 286: 205-212, 1972

4) Cho KR, Olson JL, Epstein JI: Primitive rhabdomyosarcoma presenting with diffuse bone marrow involvement: an immunohistochemical and ultrastructural study. Mod Pathol 1: 23-28, 1988

5) De la Serna FJ, Martinez MA, Valdes MD, Hornedo J, Mestre MJ, Morales JM: Rhabdomyosarcoma presenting with diffuse bone marrow involvement, hypercalcaemia and renal failure. Med Pediatr Oncol 16: 123-127, 1988

6) Douglass EC, Shapiro DN, Valentine M, Rowe ST, Carroll AJ, Raney RB, Ragab AH, Abella SM, Parham DM: Alveolar rhabdomyosarcoma with the $t(2 ; 13)$ : cytogenetic findings and clinicopathologic correlations. Med Pediatr Oncol 21: 83-87, 1993 
7) Ectubanas E, Peiper S, Stass S, Green A: Rhabdomyosarcoma presenting as disseminated malignancy from an unknown primary site: a retrospective study of ten pediatric cases. Med Pediatr Oncol 17: 39-44, 1989

8) Enzinger FM, Weiss SW: Soft Tissue Tumors, ed 3. St Louis, Mosby, 1995, pp 539-577

9) Fitzmaurice RJ, Johnson PRE, LiuYin JA, Freemont AJ: Rhabdomyosarcoma presenting as "acute leukemia." Histopathology 18: 173-175, 1991

10) Hayashi $Y$, Kikuchi F, Oka T, Itoyama S, Mohri N, Usuki K, Takaku F, Murakami T, Saitoh Y, Urano Y: Rhabdomyosarcoma with bone marrow metastasis simulating acute leukemia. Report of two cases. Acta Pathol Jpn 38: 789-798, 1988

11) Henderson DW, Raven JL, Pollard JA, Walters MNI: Bone marrow metastases in disseminated alveolar rhabdomyosarcoma: case report with ultrastructural study and review. Pathology 8: 329-341, 1976

12) Huntrakoon M, Callaway LA, Vergara GG: Systemic rhabdomyosarcoma presenting as leukemia: case report with ultrastructural study and reviews. J Surg Oncol 35: 259-265, 1987

13) Morandi S, Manna A, Sabattini E, Porcellini A:
Rhabdomyosarcoma presenting as acute leukemia. J Pediatr Hematol Oncol 18: 305-307, 1996

14) Nunez C, Abboud SL, Lemon NC, Kemp JA: Ovarian rhabdomyosarcoma presenting as leukemia. Case report. Cancer 52: 297-300, 1983

15) Putti MC, Montaldi A, D'Emilio A, Sainati L, Milanesi C, Stella M, Zanesco L, Basso G: Unusual presentation of rhabdomyosarcoma: report of two cases with immunological, ultrastructural and cytogenetical studies. Haematologica 76: 368-374, 1991

16) Ruymann FB, Newton WA, Ragab AH, Donaldson MH, Foulkes M: Bone marrow metastases at diagnosis in children and adolescents with rhabdomyosarcoma. A report from the intergroup rhabdomyosarcoma study. Cancer 53: 368-373, 1984

Address reprint requests to: T. Haisa, M.D., Department of Neurosurgery, International Medical Center of Japan, 1-21-1 Toyama, Shinjuku-ku, Tokyo 1628655, Japan. 